\title{
Sarcoidosis in the Setting of Idiopathic Chronic Bronchiolitis with Airway Colonization from $P$. aeruginosa: Treatment with Low-Dose Macrolides
}

\author{
Rocco Trisolini ${ }^{1}$, Alessandra Cancellieri ${ }^{2}$, Daniela Paioli ${ }^{1}$, Marcellino Burzi ${ }^{3}$, \\ Paolo Orlandi ${ }^{3}$ and Marco Patelli ${ }^{1}$
}

\begin{abstract}
It has long been suggested that infectious agents may trigger sarcoidosis through their infectious or antigenic properties. A patient with airway colonization from P. aeruginosa in the setting of idiopathic bronchiolitis (IB) mimicking diffuse panbronchiolitis (DPB) developed sarcoidosis. Impressive clinical and radiological improvement of both bronchiolitis and sarcoidosis features was achieved with a one-year treatment with low-dose erythromycin, thus suggesting a possible link between the two conditions in this specific case. Pathogenic hypotheses and therapeutic implications are specifically discussed.
\end{abstract}

Key words: bronchiolitis, bronchoscopy, diffuse panbronchiolitis, macrolides, P. aeruginosa, sarcoidosis

(Inter Med 47: 537-542, 2008)

(DOI: 10.2169/internalmedicine.47.0675)

\section{Introduction}

The definition "idiopathic bronchiolitis (IB) mimicking diffuse panbronchiolitis (DPB)" refers to a group of chronic idiopathic small airway diseases with clinical and radiological findings very much like those of DPB $(1,2)$. In the original series, described by Poletti et al in 2003, the patients had productive cough, exertional dyspnea, obstructive ventilatory impairment, marked neutrophilia in the BAL fluid, and findings consistent with diffuse bronchiolar involvement (centrilobular nodules, tree in bud opacities, bronchiectasis, peripheral oligoemia) on HRCT (1). Most of the patients were colonized either by gram positive bacteria or, at a later stage, by gram negative bacteria such as $P$. aeruginosa (1). As compared to DPB patients, however, they lacked diffuse pansinusitis and the typical histological pattern (1). Furthermore, long-term, low-dose, macrolide treatment, which has changed the natural history of DPB, proved beneficial to a lesser degree for IB than for DPB (1-4).

In the case herein reported, a patient with airway colonization by $P$. aeruginosa in the setting of IB mimicking
DPB, developed sarcoidosis. Curiously, impressive improvement of both bronchiolitis and sarcoidosis was observed with a 1-year, low-dose, macrolide treatment, which lead us to speculate a possible etiologic and pathogenic link between the two conditions in this specific case.

\section{Case Report}

A 66-year-old non-smoking man patient was referred to our unit in May 2001 for bronchoscopy. His past medical history was remarkable for recurrent episodes of bronchitis with sputum production since childhood, mostly treated with antibiotics. In the last three years he was prescribed inhaled corticosteroids and $\beta-2$ agonists due to worsening of his symptoms with dyspnoea at minimum effort, and productive cough. Eventually, long-term oxygen therapy was also instituted due to the occurrence of hypoxemia at rest.

On admission he was afebrile, and had normal vitals, EKG and echocardiogram. Clinical examination revealed coarse crackles at the base of both lung fields. Neither symptoms nor signs related to joint or bowel diseases were evident in his past and present medical history. Pulmonary

${ }^{1}$ Thoracic Endoscopy and Pulmonology Unit, Maggiore Hospital, Bologna, Italy, ${ }^{2}$ Pathology Unit, Maggiore Hospital, Bologna, Italy and ${ }^{3}$ Radiology Unit, Maggiore Hospital, Bologna, Italy

Received for publication October 16, 2007; Accepted for publication December 20, 2007

Correspondence to Dr. Rocco Trisolini, rocco.trisolini@ausl.bologna.it 


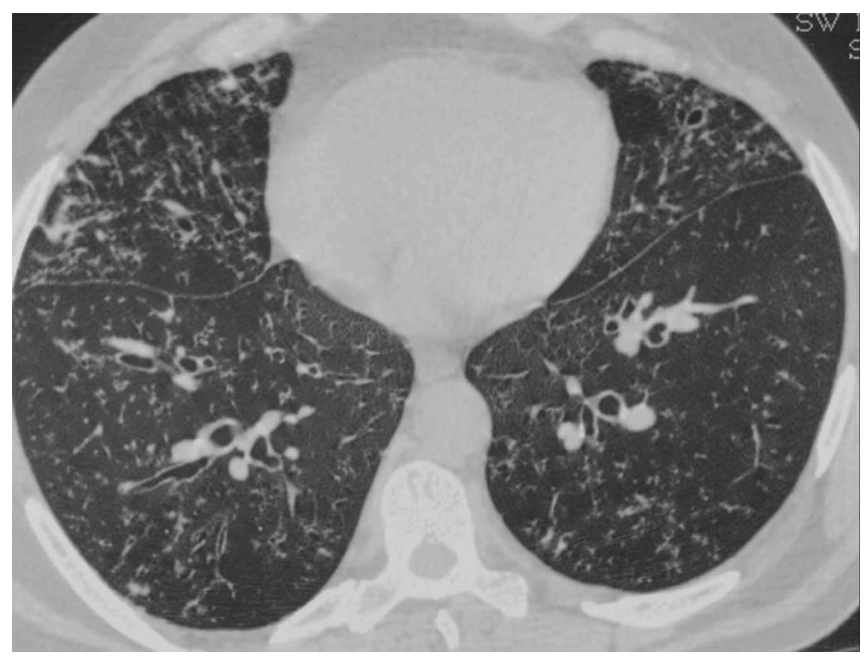

Figure 1. HRTC scan showing diffuse centrilobular nodules, tree in bud pattern, and thickening of bronchobronchiolar walls, as well as clean bronchiectasis and bronchiolectasis.

function test (PFT) showed severe obstructive ventilatory defect $\left(\mathrm{FEV}_{1} 32 \%\right.$ of predicted; $\left.\mathrm{FEV}_{1} / \mathrm{FVC} 40 \%\right)$; a challenge with short-acting $\beta-2$ agonists did not provide any improvement. Blood gas values while breathing room air showed severe hypoxemia $\left(\mathrm{PaO}_{2} 51 \mathrm{mmHg}\right)$. Laboratory findings were remarkable for increase of ESR $(33 \mathrm{~mm} / 1 \mathrm{~h})$, circulating immune complexes (CIC) $(5.5 \mu \mathrm{g} / \mathrm{ml}-$ n.v. $<1.5 \mu \mathrm{g} / \mathrm{ml})$, and Ca 19.9 serum levels (76 U/ml - n.v. <33 U/ml). Rheumathoid factor testing proved negative. HRCT-scan showed findings very much like those observed in DPB (Fig. 1): diffuse centrilobular opacities, tree in bud pattern, bronchiectasis and bronchiolectasis, peripheral oligoemia and, on expiratory scans, air trapping. Neither pleural nor mediastinal abnormalities were observed. Cytological examination of BAL-fluid showed a huge increase of the neutrophil percentage (90\%), and cultures grew P. aeruginosa. Sweat test and genetic screening for cystic fibrosis, as well as serum levels of $\mathrm{Ig}-\mathrm{E}$ and $\mathrm{Ig}-\mathrm{G}$ against A. fumigatus proved negative. A diagnosis of IB mimicking DPB was proposed. After two antibiotic courses based on the drug susceptibility test did not provide any clinical-radiological improvement, longterm, low-dose, macrolide therapy (erythromycin $600 \mathrm{mg} /$ day) was prescribed, but the patient's compliance to the treatment was poor. Over the following year, he complained of increasing production of purulent sputum from which $P$. aeruginosa was constantly cultured (frequently with quantitative cut-off values $\geq 10^{6} \mathrm{cfu} / \mathrm{ml}$ ), and his medical conditions progressively declined. Blood oxygen values at rest while breathing room air were as high as $49 \mathrm{mmHg}$. Chest CT scan showed marked worsening of bronchiolitic features along with the presence of large hilar and mediastinal lymphadenopathy (Fig. 2a, b). Tuberculin skin test was negative, whereas increase of angiotensin-converting enzyme (s-ACE) (107 U/1 - n.v. 8-52 U/l), Ca 19.9 (134 U/ml - n.v. <33 U/ $\mathrm{ml})$, and CIC $(8.8 \mu \mathrm{g} / \mathrm{ml}-$ n.v. $<1.5 \mu \mathrm{g} / \mathrm{ml})$ was observed. Ophthalmology examination as well as cardiologic work-up were negative. At this time steps were taken to perform rigid bronchoscopy with bronchial washing, transbronchial needle aspiration (TBNA) of a subcarinal lymph node, and transbronchial lung biopsy (TBLB). At pathologic examination, TBNA yielded lymphocytes-rich smears with nonnecrotizing microgranulomas (Fig. 3a). TBLB showed sparse, non-necrotizing microgranulomas consisting of wellformed aggregates of giant cells and epithelioid macrophages (Fig. 3b). Nodular collections of peribronchiolar lymphocytes consistent with cellular bronchiolitis (Fig. 3c), and the presence of endobronchiolar muco-purulent exudate consistent with suppurative bronchiolitis were also evident. Neither acid-fast bacilli nor other microbes were seen in both TBNA and TBLB samples. Direct examination of bronchial washing was negative for mycobacteria and fungi, whereas cultures grew $P$. aeruginosa. A diagnosis of sarcoidosis superimposed to IB mimicking DPB was proposed. Remarkably, no cases of sarcoidosis or small airway disease run in the family of the patient. As steroid treatment was deemed potentially harmful by considering the airway colonization by $P$. aeruginosa, low-dose macrolide therapy (erythromycin $600 \mathrm{mg} /$ day) was initiated. In two months the patient reported a marked clinical improvement characterized by reduction of cough, sputum production, and dyspnoea. By the sixth month after treatment initiation, $P$. aeruginosa was not isolated anymore on sputum cultures. At 1-year follow-up, the clinical improvement was stable and was associated with improvement of blood oxygen values at rest $\left(\mathrm{PaO}_{2} 60 \mathrm{mmHg}\right)$, normalization of many laboratory parameters including s-ACE (22 U/1 - n.v. 8-52 U/l), and Ca 19.9 (17.2 U/ml - n.v. $<33 \mathrm{U} / \mathrm{ml})$, and impressive reduction of radiological abnormalities. CT scan, in fact, showed marked improvement of the bronchiolitis-related findings, as well as normalization of hilar and mediastinal lymph node size (Fig. 4a, b). PFT values did stabilize $\left(\mathrm{FEV}_{1}\right.$ 32.9\%; $\mathrm{FEV}_{1} / \mathrm{FVC} 52 \%$ ) (Fig. 5a, b). After a further 2-year treatment with the same low-dose macrolide regimen, the clinical, functional and radiological findings are stable, and eradication of $P$. aeruginosa from the airways Has been confirmed.

\section{Discussion}

We report on a case of sarcoidosis occurring in a patient with IB mimicking DPB. The diagnosis of sarcoidosis was based, in this case, on the pathologic findings of broncoscopic samples (obtained both from the lung and mediastinal lymph nodes), on the chest CT-findings (hilar and mediastinal lymphadenopathy), and on the laboratory data (increase of s-ACE) $(5,6)$. Alternative diagnosis such as occupational and environmental exposures possibly related to granulomatous lung disease, fungal and mycobacterial infection, and sarcoid-like local reaction to a malignancy, were taken into account and reliably ruled out (6). We took into account the possibility that the histologic findings of the present case were an expression of the sarcoid-like reaction to chronic $P$. aeruginosa infection, but ruled it out based on 

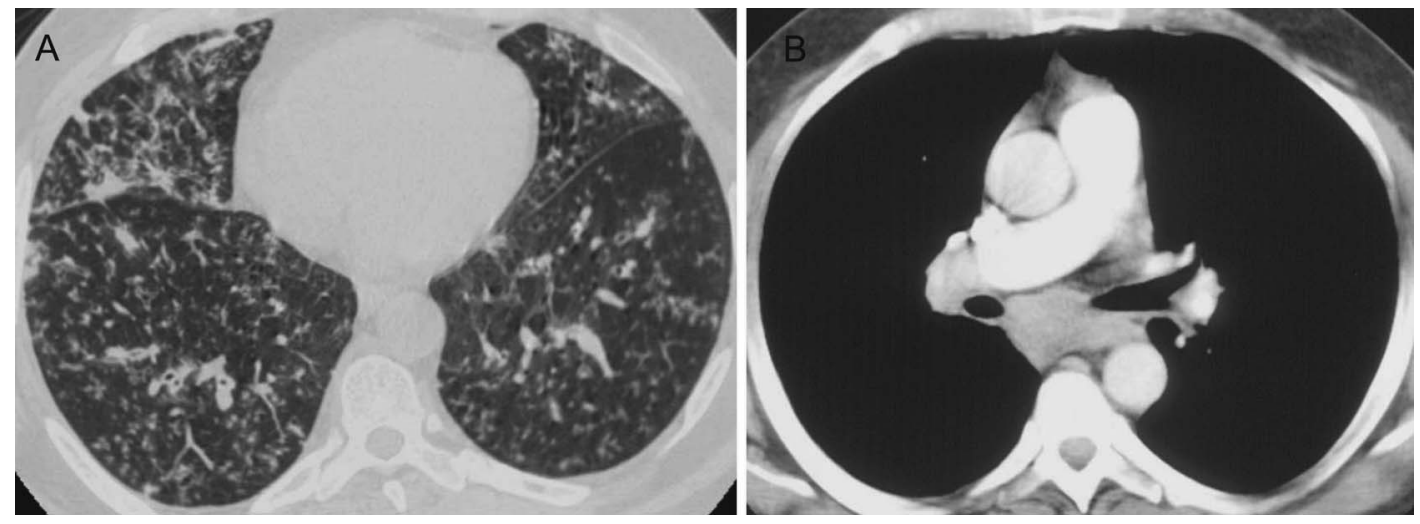

Figure 2. a) HRCT scan at the same level of Fig. 1 showing marked worsening of bronchiolitic features. Marked increase of the profusion of centrilobular nodules and tree in bud opacities is evident. b) Contrast-enhanced CT scan at the level of the tracheal carina showing enlarged subcarinal and right hilar lymph nodes.
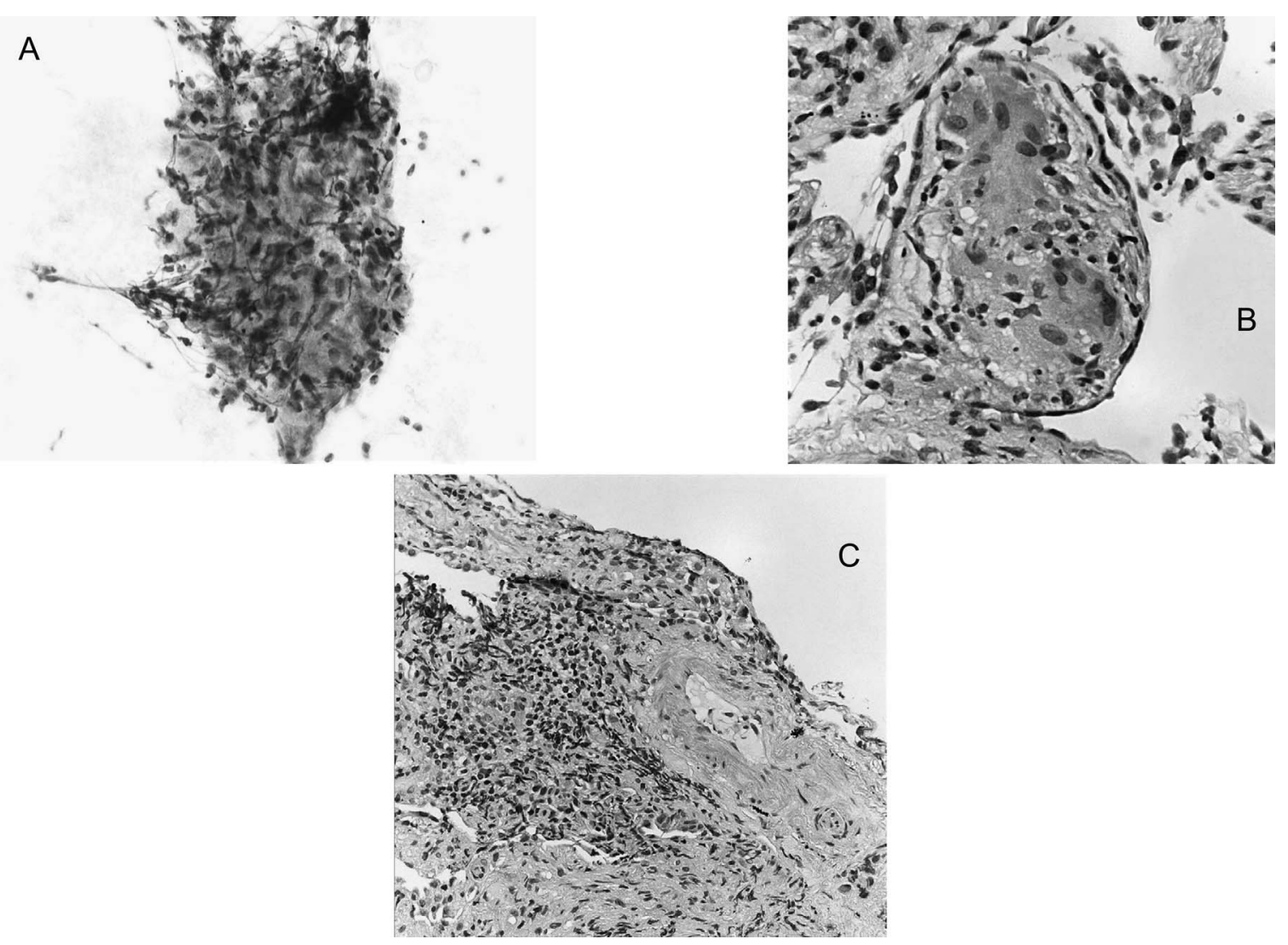

Figure 3. a) An isolated, non necrotizing microgranuloma, in the cytological sample obtained with TBNA (Papanicolaou, 400x). b) A small, non necrotizing granuloma composed of multinucleated giant cells and macrophages (Hematoxylin and Eosin staining, 400x). c) A nodular collection of peribronchiolar lymphocytes (Hematoxylin and Eosin staining, 200x).

the following two reasons: a) granulomas have never been reported among the histologic findings of pseudomonas infection in the human $(7,8)$; b) granulomas were not limited to the lung but were also found in the mediastinal lymph nodes.

Although no literature reports exist, to the best of our knowledge, on the association between IB mimicking DPB (or DPB itself) and sarcoidosis, the impressive improvement of both bronchiolitis and sarcoidosis-related features (normalization of s-ACE and hilar/mediastinal lymph node size) after low-dose, macrolide treatment, lead us to speculate on a relationship between the two pathologic entities in this 

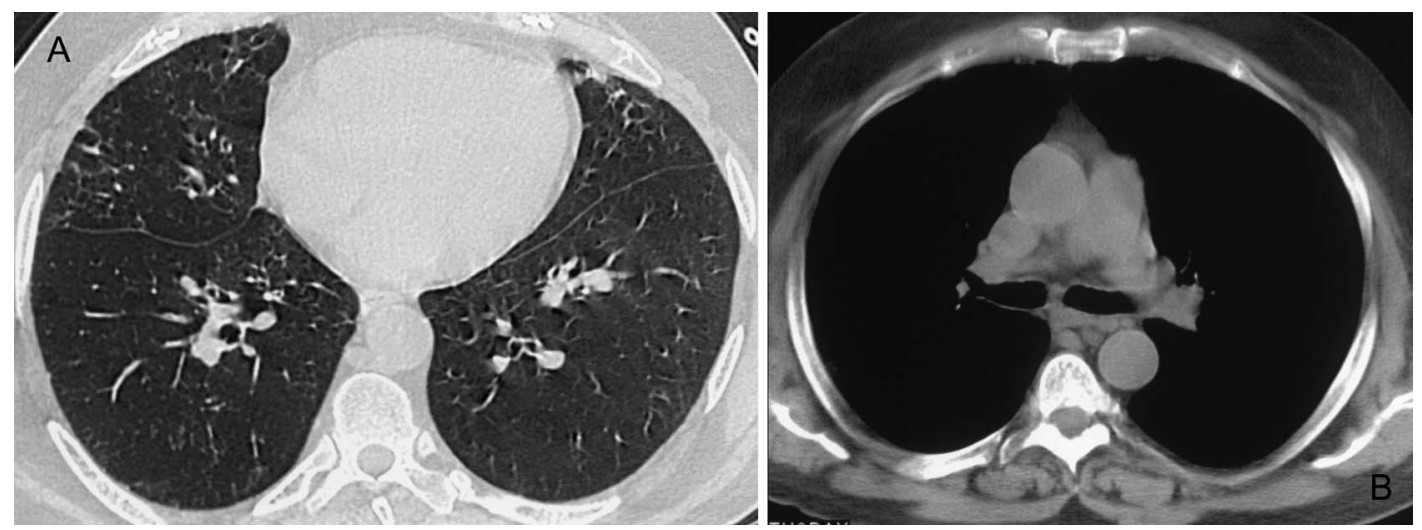

Figure 4. a) HRCT scan at the same level of Figs. 1 and 2 showing impressive improvement of bronchiolitic features, with marked reduction of centrilobular nodules and tree in bud opacities. b) CT scan at the level of the tracheal carina showing normalization of the subcarinal lymph node size.
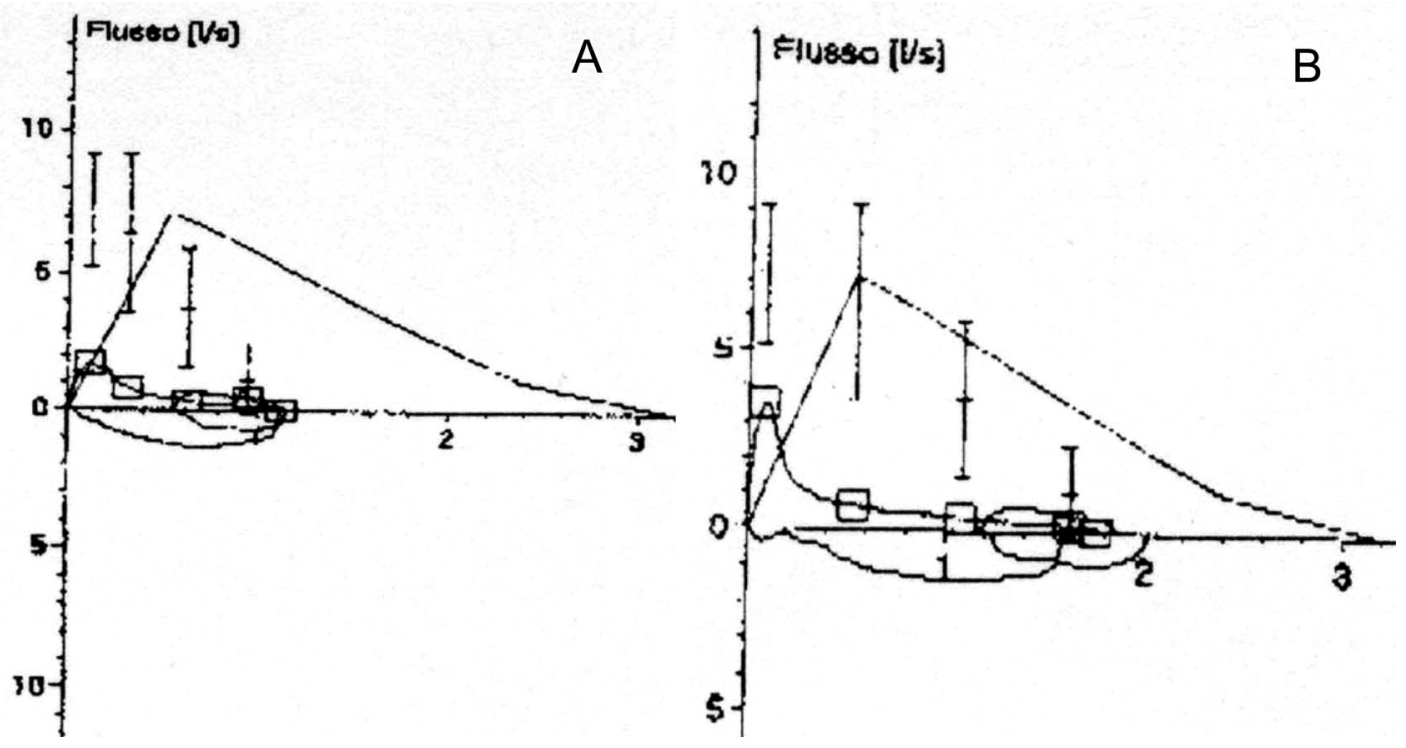

Figure 5. a) Flow-volume curve before macrolide treatment. b) Flow-volume curve after two years of macrolide treatment.

specific case.

A possible missing link between the two conditions might have been $P$. aeruginosa. It is a largely shared opinion that sarcoidosis is the result of a strong polygenic predisposition triggered by one or more microbes acting through their infectious or antigenic properties $(6,9-11)$. The role of infectious agents is supported by evidence such as spatial, seasonal, and familial clustering, as well as isolation of footprint of candidate organisms (proteins or nucleic acids) from patient samples $(6,9-11)$. Although mycobacteria and propionibacteria have emerged as the most likely candidates, no single agent has been identified as definite culprit in spite of considerable research efforts $(6,9-11)$. Curiously, $P$. aeruginosa was responsible for the formation of pulmonary granulomas in an experimental rabbit model of chronic bronchiolitis DPB-like induced by intratracheal inoculation of the pathogen (12). What is more, treatment with erythromycin resulted in a significant inhibition of neutrophil recruit- ment in the lung, a reduction in the BAL levels of proinflammatory mediators (especially TNF- $\alpha$ and IL8) thought to be critical to granuloma formation/persistence, and a reduction in size of granulomatous lung lesions by $50 \%$ on day 14 (12). Both the disease context and the beneficial effect of erythromycin treatment in the above animal model are very much like those observed in the present case, although we did not proceed (for ethical reasons) to a histological follow-up due to the impressive improvement of clinical and radiological findings. If we accept as plausible the hypothesis that $P$. aeruginosa had a role in the onset of sarcoidosis in this case, then the beneficial effect of lowdose erythromycin can be explained by several mechanisms $(13,14)$.

It has been demonstrated that macrolides, when given at sub-MIC concentration, do not inhibit $P$. aeruginosa production of type IV pili, but they impede their assembly on the surface of the bacterium (13). Among other important conse- 
quences (impaired twitching motility and adhesion capacity to epithelial cells) on its virulence (14-16), the absence of pili at the surface of $P$. aeruginosa can modify antigenic properties that might have been important in the pathogenic events leading to sarcoidosis in the present case.

Low-dose macrolides may also alter the formation of the Pseudomonas biofilm, a highly ordered microbial community enmeshed in a carefully sculpted matrix, designed to protect the bacterium from phagocytosis, antibiotics, the ciliary action of the respiratory tract, opsonizing antibodies, and complement (17). The alteration of structure/function of the biofilm may enhance the clearance of bacteria by the patient's own pulmonary host defence, and/or may result in more efficient access of antipseudomonal antibiotics (13, 14). This mechanism might have contributed to eradication of $P$. aeruginosa in the present case, thus helping remove from the airway a possible stimulus for granuloma formation.

Beyond their direct effect on virulence determinants of $P$. aeruginosa, low-dose macrolides have been shown to influence cytokine/chemokine production by several cell types, and to alter polymorphonuclear cell functions in vitro and ex vivo (14). Macrolide-induced suppression of IL-1 $\beta$, IL-6, IL8 , TNF- $\alpha$ from several cell types challenged with different stimuli (eg. proinflammatory cytokines, LPS, bacteria) has been demonstrated in vitro and ex vivo (14). It is of note that high levels of IL- 8 and TNF- $\alpha$, whose production is inhibited by macrolides, have been associated with chronic disease and long-term therapy in the setting of sarcoidosis $(18-20)$. It is therefore possible that the reduction of the lev- els of the above cytokines might have been another factor contributing to the improvement of sarcoidosis in the present case.

Nonetheless, different interpretations on the relationship between IB mimicking DPB and sarcoidosis observed in the present case should also be considered: 1) in view of the lack of literature reports on the association between sarcoidosis and pathologic conditions characterized by chronic neutrophilic airway inflammation and $P$. aeruginosa colonization (IB mimicking DPB, DPB itself, cystic fibrosis, chronic bronchiectasis), which would be expected if $P$. aeruginosa were a common trigger for sarcoidosis, it is obviously possible that the association observed in our case was a chance one. If this were the case, it is still possible that macrolides contributed to the improvement of sarcoidosis by inhibiting the production of cytokines associated with a prolonged disease course, a drug activity which is not limited to $P$. aeruginosa infection $(14,18-20)$; 2$)$ It is possible that the development of sarcoidosis preceded that of chronic infection from $P$. aeruginosa. As sarcoidosis may involve the small airways, bronchiolar obstruction might have been a predisposing factor to $P$. aeruginosa infection/colonization.

In conclusion, we hypothesize that $P$. aeruginosa might have triggered sarcoidosis in our patient with IB mimicking DPB. Long-term, low-dose, macrolide treatment might have been beneficial for sarcoidosis by modifying the surface antigenic properties of $P$. aeruginosa, by facilitating eradication of the pathogen from the airways through alteration of its biofilm, and by inhibiting the production of cytokines crucial to formation/persistence of granulomas.

\section{References}

1. Poletti V, Chilosi M, Trisolini R, et al. Idiopathic bronchiolitis mimicking diffuse panbronchiolitis. Sarcoidosis Vasc Diffuse Lung Dis 20: 62-68, 2003.

2. Kadota J, Mukae H, Mizunoe S, et al. Long-term antibiotic therapy in the treatment of chronic small airway disease clinically mimicking diffuse panbronchiolitis. Intern Med 44: 200-206, 2005.

3. Poletti V, Chilosi M, Casoni G, Colby TV. Diffuse panbronchiolitis. Sarcoidosis Vasc Diffuse Lung Dis 21: 94-104, 2004.

4. Kudoh S, Azuma A, Yamamoto M, Izumi T, Ando M. Improvement of survival in patients with diffuse panbronchiolitis treated with low-dose erythromycin. Am J Respir Crit Care Med 157: 1829-1832, 1998.

5. Gal AA, Koss MN. The pathology of sarcoidosis. Curr Opin Pulm Med 8: 445-451, 2002.

6. Hunninghake GW, Costabel U, Ando M, et al. Statement on sarcoidosis. Sarcoidosis Vasc Diffuse Lung Dis 16: 149-173, 1999.

7. Meier FA. Pseudomonas aeruginosa. In: Pathology of Infectious Diseases. Connor DH, Chandler FW, Manz HJ, et al, Eds. Appleton \& Lange, Stamford, 1997: 647-656.

8. Pseudomonas aeruginosa. In: Non-neoplastic disorders of the lower respiratory tract. Travis WD, Colby TV, Koss MN, Rosadode-Christenson ML, Muller NL, King TE, Eds. AFIP, ARP, 2002; 550-553.

9. McGrath DS, Goh N, Foley PJ, du Bois RM. Sarcoidosis: genes and microbes - soil or seed? Sarcoidosis Vasc Diffuse Lung Dis 18: 149-164, 2001.
10. Baughman RP, Lower EE, du Bois RM. Sarcoidosis. Lancet 361: 1111-1118, 2003.

11. Newman LS, Rose CS, Bresnitz EA, et al. A case control etiologic study of sarcoidosis: environmental and occupational risk factors. Am J Respir Crit Care Med 170: 1324-1330, 2004.

12. Takahashi T, Suga M, Matsukawa A, et al. Erythromycin attenuates an experimental model of chronic bronchiolitis via augmenting monocyte chemoattractant protein-1. Eur Respir J 17: 360367, 2001.

13. Wozniak DJ, Keyser R. Effects of subinhibitory concentrations of macrolide antibiotics on Pseudomonas aeruginosa. Chest 125: 62 S-69S, 2004.

14. Schultz MJ. Macrolide activities beyond their antimicrobial effects: macrolides in diffuse panbronchiolitis and cystic fibrosis. J Antimicrob Chemother 54: 21-28, 2004.

15. Semmler A, Whitchurch CB, Mattick JS. A re-examination of twitching motility in Pseudomonas aeruginosa. Microbiology 145 : 2863-2873, 1999.

16. Sheth HB, Lee KK, Wong WY, et al. The pili of Pseudomonas aeruginosa strains PAK and PAO bind specifically to the carbohydrate sequence bGalNAc (1-4) beta Gal found in glycosphingolipids asialo-GM1 and asialo-GM2. Mol Microbiol 11: 715-723, 1994.

17. Costerton JW, Stewart PS, Greenberg EP. Bacterial biofilms: a common cause of persistent infections. Science 284: 1318-1322, 1999.

18. Ziegenhagen MW, Benner UK, Zissel G, Zabel P, Schlaak M, 
Muller-Quernheim J. Sarcoidosis: TNF-alpha release from alveolar macrophages and serum level of sIL $2 \mathrm{R}$ are prognostic markers. Am J Respir Crit Care Med 156: 1586-1592, 1997.

19. Baughman RP, Keeton D, Lower EE. Relationship between interleukin-8 and neutrophils in the BAL fluid of sarcoidosis. Sarcoidosis 11: S217-S220, 1994.
20. Baughman RP, Strohofer SA, Buchsbaum J, Lower EE. Release of tumor necrosis factor by alveolar macrophages of patients with sarocidosis. J Lab Clin Med 115: 36-42, 1990.

(C) 2008 The Japanese Society of Internal Medicine http://www.naika.or.jp/imindex.html 